\title{
FREE VASCULARISED FIBULAR TRANSPLANT FOR REPLACEMENT OF THE LOWER RADIUS
}

\author{
ROBERT W. H. PHO \\ From University of Singapore, Department of Orthopaedic Surgery, Singapore General Hospital
}

An operation is described in which a microvascular technique was used to transfer a living fibula, with its vascular pedicle intact, to replace the lower end of the radius after massive resection for giant-cell tumour. Angiography carried out six weeks later showed that the grafted bone was viable. Six months after operation the transplanted fibula showed no osteoporosis or bone resorption and bony union at the junction of host and graft.

The use of the proximal fibula to replace the lower end of the radius in cases of giant-cell tumours is well established (Lawson 1952; Goldenberg, Campbell and Bonfiglio 1970; Pitcock 1971). The main drawback of this technique has been that the fibular graft, being a long cortical bone placed in a relatively avascular bed, nearly always became osteoporotic, progressively absorbed and was only slowly incorporated. Despite prolonged immobilisation, the patient often ended up with a stress fracture, a deformity, and delayed or

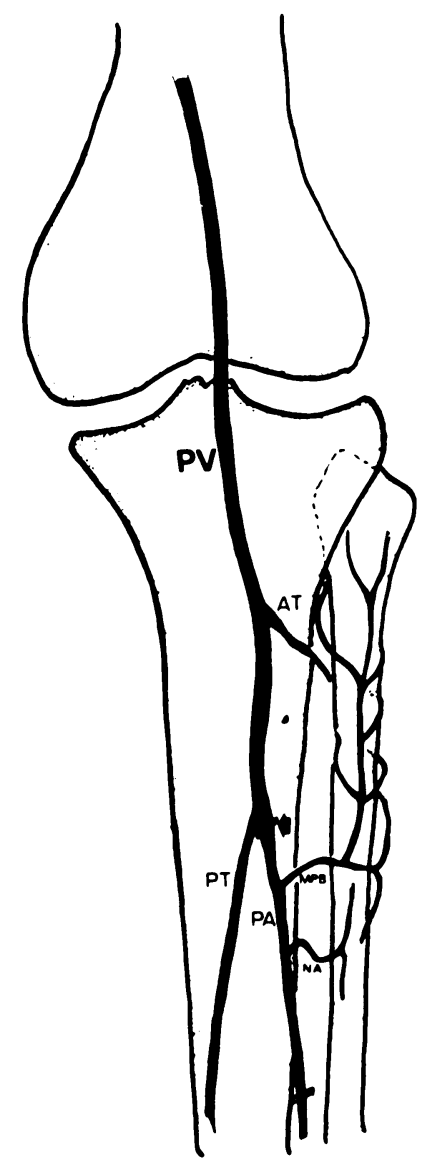

non-union (Stewart and Richardson 1952; Tuli 1972; Pho and Balachandran 1978). We have attempted to overcome these problems by using a microvascular technique to transfer the fibula with its peroneal vascular pedicle as a living bone graft anastomosed to the radial artery and a cutaneous vein.

Blood supply to fibula. The proximal half of the fibula receives its blood supply mainly from the peroneal artery through the main nutrient artery (Crock 1967) and numerous segmental vessels (Taylor, Miller and Ham 1975). These branches, which provide the blood supply for muscle and periosteum, form multiple arcades around the fibula and are interconnected by vessels running parallel to the long axis of the bone. Our studies of the blood supply to freshly dissected fibulae confirmed these findings (Pho 1979; Figs. 1 and 2).

\section{CASE REPORT}

A twenty-three-year-old woman presented with a painful swelling over the lower end of the right radius. Radiographs showed an expanding lytic lesion consistent with the diagnosis of giant-cell tumour, which was confirmed by histological examination.

A free-living fibular replacement for the lower end of the radius with microanastomosis of the vascular pedicle was decided in view of

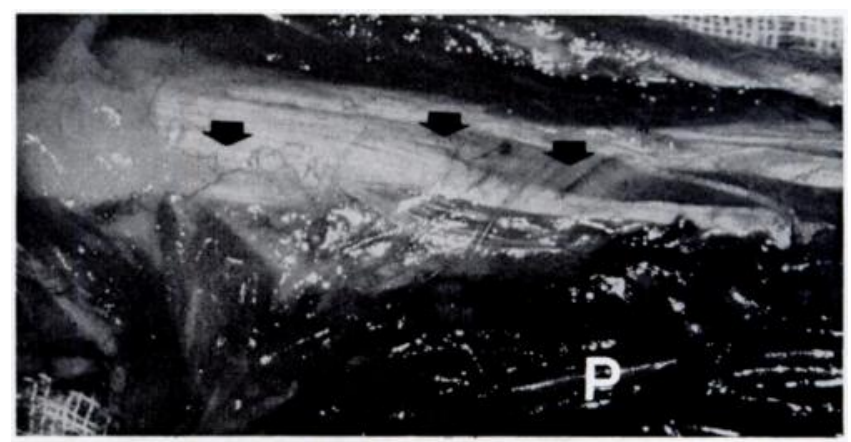

Fig. 2

Freshly dissected specimen of proximal half of fibula with 1 centimetre muscle sleeve and intact peroneal vascular pedicle $(P)$. Methylene blue had been injected into the peroneal artery. Arrows indicate multiple branches supplying the surrounding muscles and the fibula.

R. W. H. Pho, M.B.B.S.(Syd.), F.R.C.S., University of Singapore, Department of Orthopaedic Surgery, Singapore General Hospital, Outram Road, Singapore 3. 


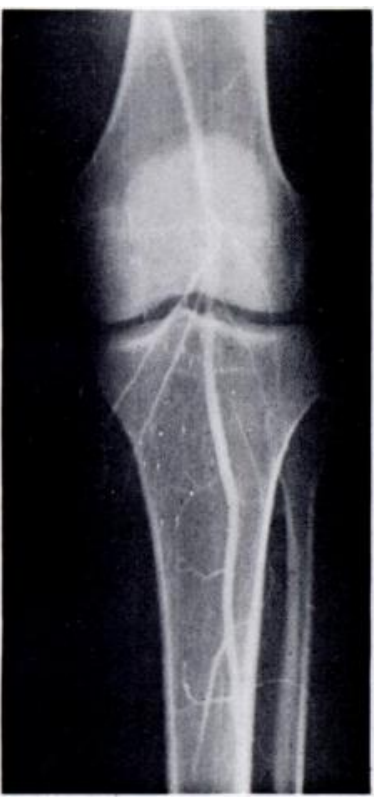

Fig. 3

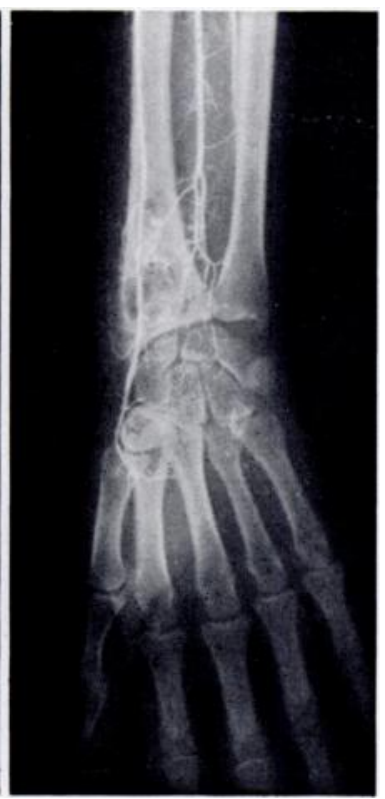

Fig. 4
Figure 3-Angiography of the leg to map out the blood supply to the fibula. Figure 4-The vascular pattern of the right forearm outlined for selection of suitable artery for anastomosis.

our previous unsatisfactory experiences in seven similar cases treated by the conventional method of reversed proximal fibular grafts (Pho and Balachandran 1978).

The affected right forearm and contralateral leg were first subjected to angiography to outline the vascular anatomy in the limbs (Figs. 3 and 4). It was essential to have this information before operation to determine the level of osteotomy of the fibula and to select the donor vessel in the forearm for anastomosing to the peroneal vessel.

\section{OPERATIVE TECHNIQUE}

With the patient under general anaesthesia, two teams operated simultaneously. One team exposed the radius through anterior lazy-S incision. The flexor muscles were retracted and the radial artery identified. Two dorsal cutaneous veins were identified and preserved. Extensor carpi radialis longus and brevis were freed. Pronator quadratus together with the lower end of the radius were separated from the surrounding tissue. The anterior capsule of the wrist joint was transected with minimal preservation of capsular ligament. Fifteen centimetres of the lower end of radius together with the tumour and pronator quadratus with intact periosteum were removed.

The second team exposed the upper end of the fibula along its posterior border. The incision was curved medially and upwards at the level of the knee joint. The lateral popliteal nerve and its branches were identified, and a sling was used to retract the nerve anteriorly. The lateral head of the gastrocnemius muscle was retracted medially right down to the capsule of the knee joint when the popliteal neurovascular bundle was identified. The attachment of soleus to the fibula was identified and divided, keeping a 1 centimetre sleeve of muscle insertion over the fibula on the posteromedial side to enable preservation of muscular periosteal vessels.

The peroneal blood vessels were identified and preserved carefully along with their branches supplying the fibula. Venae comitantes from the muscle and the fibula were also identified and preserved. The dissection was continued to about 17 centimetres below the head of the fibula. At this level the flexor hallucis longus was partly divided close to the bone. The dissection was then extended anteriorly with preservation of the sleeve of the peroneus longus, tibialis anterior and the interosseous membrane around the fibula. The anterior tibial vessels and deep peroneal nerve were carefully preserved and retracted anteriorly.

The distal fibula was transected with a Gigli saw; disarticulation of the superior tibiofibular joint was carried out and then the fibular collateral ligament was reconstructed.

The distal ends of the peroneal artery and venae comitantes were ligated and divided. The fibula was freed from all surrounding tissue with the exception of its proximal vascular pedicle, the peroneal artery and venae comitantes, which were still in continuity with the popliteal vessels (Fig. 5). The tourniquet was then released to assess the vascularity of the proximal fibula. It was evident that the fibula had been raised successfully and was viable with bright bleeding over the fibular head, the cut sleeves of muscle and the medullary cavity distally. The peroneal artery and venae comitantes were then divided at their bifurcation from the posterior tibial artery and the popliteal vein.

The fibula was shortened by a further 2 centimetres to fit in with the resected radius. Using a five-hole plate and four screws, the fibula was fixed to the radius. The screws were kept away from the nutrient artery to the fibula by careful measurement of the level of entry of nutrient vessel. The wrist was transfixed with a Kirschner wire passed from the scaphoid to the fibula. The anterior capsular ligament of the wrist was stitched to the lower end of the fibula.

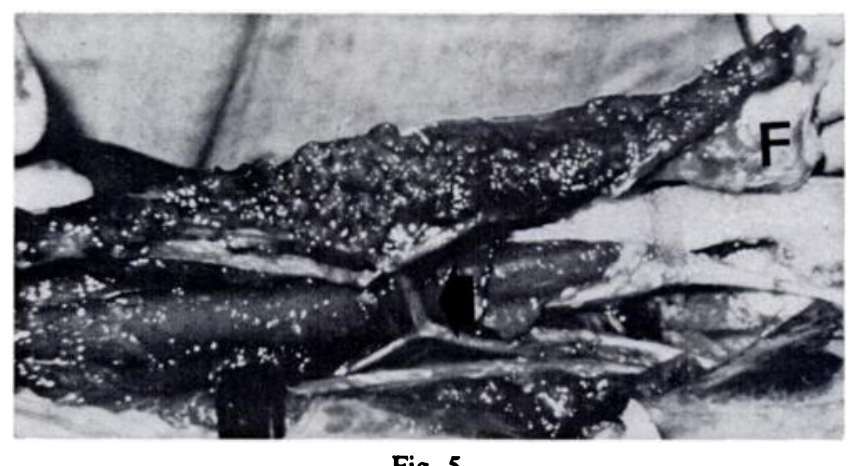

Fig. 5

Fibula (F) has been freed completely except for its vascular pedicle (arrow).

The radial artery was transected and looped around to anastomose to the proximal end of the peroneal artery (Figs. 6 and 7). Although the peroneal artery was about one-third bigger than the radial artery, a good anastomosis was achieved. Altogether there were fifteen interrupted 10/0 Ethilon sutures inserted. On completion of arterial anastomosis, there was profuse bleeding along the muscle sleeves and the periosteum of the fibular head with good return along venae comitantes. One cutaneous vein was identified and anastomosed to venae comitantes with interrupted 10/0 Ethilon sutures.

Direct skin closure was possible on the proximal part of the wound. The distal part required a split skin graft measuring 10 by 2 centimetres to cover the skin defect. Two Redivac drains were inserted over the right forearm and a long plaster back-slab was applied. After operation the patient was given 1 litre of 10 per cent dextran in normal saline daily for one week, aspirin 600 milligrams eight hourly for three weeks, and ampicillin and cloxacillin 500 milligrams of each six hourly for one week.

\section{PROGRESS}

After operation the patient was unable to flex the interphalangeal joint of her thumb, probably due to extensive dissection of the muscle origin having interfered with the blood supply to the flexor pollicis longus. It was also noted that the patient had a foot drop 
caused by traction on the lateral popliteal nerve. The wound healed uneventfully.

Arteriography was carried out six weeks after the fibular transfer. The plain radiograph showed no osteoporosis; the consistency of the fibular graft was similar to the remaining radius and ulnar bones. Bony union at the junction of graft and host was progressing and the anastomosis was patent. There was excellent arterial flow supplying the whole transferred fibula (Figs. 6 and 7). The forearm was left free for exercise.

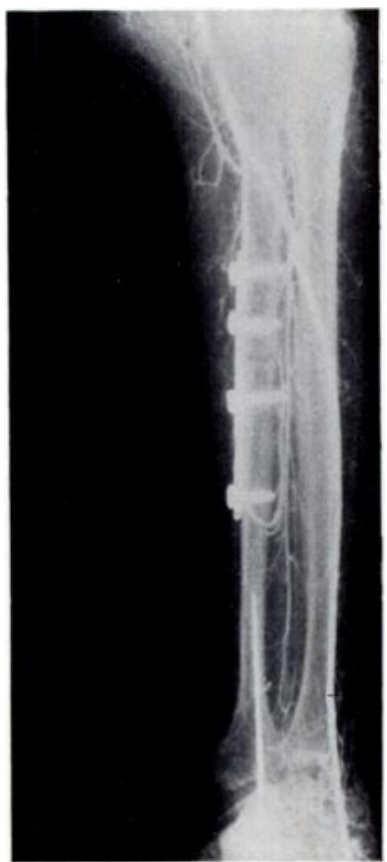

Fig. 6

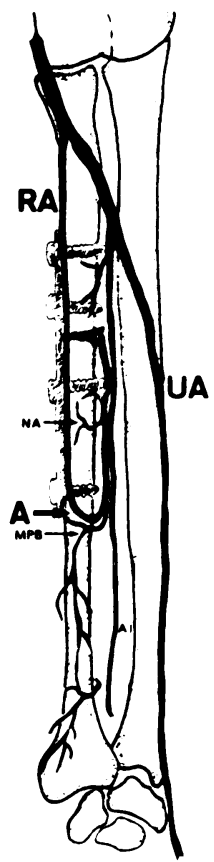

Fig. 7
Figure 6-Six weeks after fibular transfer, angiography showed patency of anastomosed vessels with clear outline of the nutrient artery and muscular periosteal vessels supplying the whole length of the fibula and its head. Note that the consistency of the transferred fibula is the same as the remaining radius and ulna. There was evidence of bony union at the junction of host and graft. Figure 7-Diagram of transferred fibula and its blood supply as shown by angiography. AI, Anterior interosseous artery; UA, ulnar artery; RA, radial artery; $\mathbf{A}$, site of anastomosis of radial artery to peroneal artery; NA. nutrient artery; MPB, muscular periosteal branches.

The action of flexor pollicis longus returned two months after operation, and by four months the lateral popliteal nerve palsy had recovered; bony union of host and graft was complete six months after fibular transplant. The patient was able to use her right hand almost normally four months after operation. She had no pain but her grip was slightly weak. She had 60 degrees of pronation and supination with 25 degrees of dorsiflexion and 40 degrees of palmar flexion at the wrist, with 25 degrees of ulnar and radial deviation.

Bone scanning, which could not be carried out until six months after operation, showed marked increase in blood supply along the transplanted fibula (Fig. 8).

\section{DISCUSSION}

Curettage, bone grafting, irradiation and resection have been used in the treatment of giant-cell tumour of the lower end of the radius (Dahlin, Cupps and Johnson 1970). Undoubtedly resection and replacement of the radius offer the best result. The proximal fibula and its head (Parrish 1966), upper tibial corticocancellous bone graft (Campbell and Akbarnia 1975) and iliac bone graft (Higinbotham and Coley 1950; Wilson and Lance 1965) have been used to replace the radius after massive resection. Although many authors using these various methods have claimed good functional results, there is one distinct common disadvantage: each method uses a large avascular bone to replace the big defect in the lower end of the radius in the forearm which is itself a relatively avascular area. Most of the bone graft will become porotic, absorbed and poorly incorporated. Invariably there are problems such as non-union, stress fracture and deformity with eventual degenerative changes at the joint between the fibula and carpal bone; wrist fusions are common; in addition, these patients will require prolonged immobilisation of the forearm and the wrist and multiple bone grafts. An attempt to use a radial prosthesis has failed (Gold 1957, 1965). The logical answer therefore was to transfer a living bone. The fibula with its vascular pedicle was ideal since the fibular head conforms to the shape of the lower end of the radius.

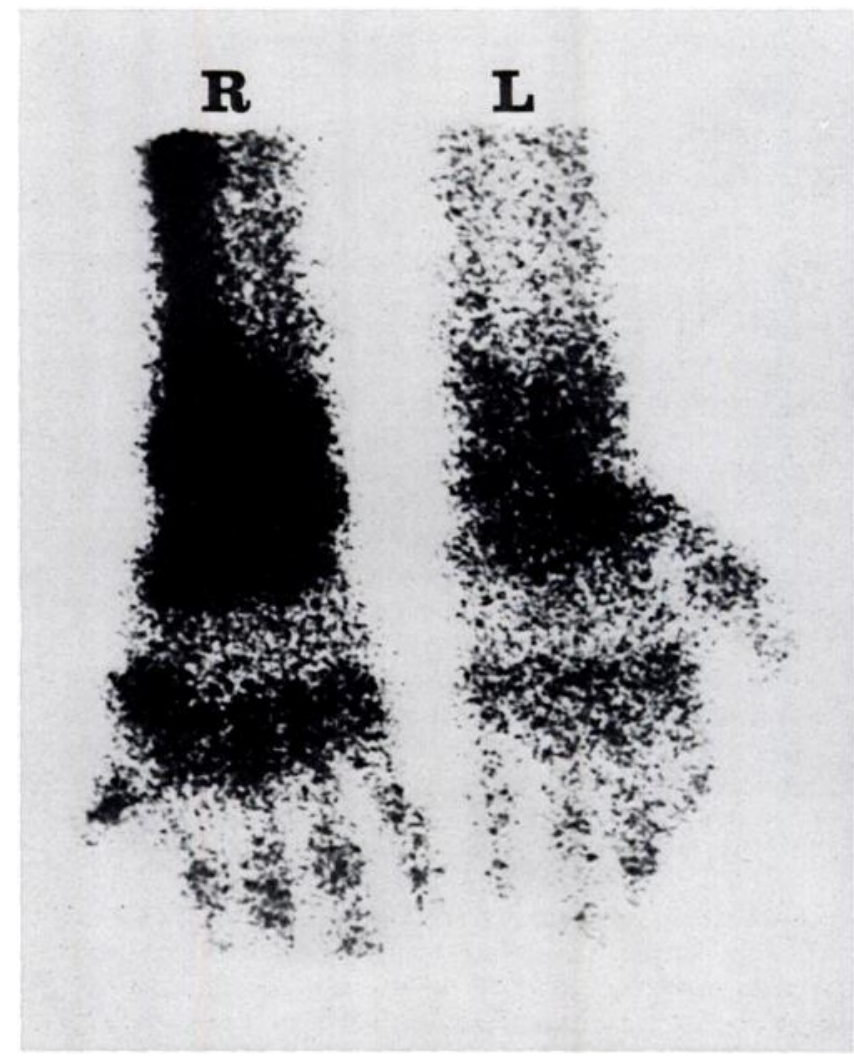

Fig. 8

A bone scan taken six months after operation showing increased blood supply of transplanted fibula (R). 
Transfer of free living bone was initiated by McCullough and Fredrickson (1973) and Östrup and Fredrickson (1974), who successfully transferred living ribs for mandible defects in dogs. Taylor and his colleagues (1975) first reported using free living fibular graft for non-union of fractured tibia in man; in their cases they used the upper end of the fibula, excluding the neck and the head because of uncertainty of the blood supply to the fibular head and to avoid disruption of the collateral ligament of the knee. In our case the proximal fibula and its head were used. The collateral ligament was detached from the fibular head and reconstructed back to the tibia. We have not encountered instability in the seven similar cases treated by conventional reversed proximal fibular grafts.

In the reported case the vascularity of the fibula and its head was confirmed by angiography six weeks after fibular transfer and microvascular suture of the peroneal artery. Anastomosis of the one artery and one vein proved to be adequate. Venous return was not a problem since there were extensive raw areas that allowed bleeding to surrounding tissue and circulatory return along the medullary cavity. It is therefore important to compress both ends of the bone if at all possible.

The conventional technique of just transferring the fibula without anastomosis of the vascular pedicle allows only slow vascularisation of the dead graft with creeping substitution. One advantage of the microvascular technique is that the transplanted living fibula behaves like a fracture of the radius; immobilisation after operation is the same as that for fracture. Non-union or bone collapse should not occur unless the anastomotic vessels are thrombosed. It is too early at this stage to state whether or not the technique will prevent degenerative changes at the reconstructed joint.

However, there are certain factors which one must consider when using this technique. It is a long operation (eight hours in this case) and requires extensive angiographic studies of vascular pattern in the limbs. If the peroneal vessels bifurcates low down and consequently the nutrient artery pierces the fibula almost halfway down the bone, as in this case, the fibular graft will have to be long and therefore the resection of the radius will be more extensive in comparison with the conventional method. The level where the nutrient vessels enter the fibula must be carefully mapped out to avoid damaging them when fixing the bone with the screws. The extensive resection of radius may affect the flexor muscles in the forearm. The possible morbidity over the donor area is relatively minor. Although two major vessels are sacrificed, the disabilities should be minimal provided thorough angiographic studies are done before operation.

I wish to thank Mr S. S. Moorthy and Mr S. H. Tow for the photographs, and Miss Janet Soh for her secretarial help; Professor P. B. Chacha, Mr K. Q. Yeo and Mr Eddie Tay for assisting me in the operation; Dr Lenny Tan for the angiography; and Dr Chang Chin Hian for the bone scan.

\section{REFERENCES}

Campbell, C. J., and Akbarnia, B. A. (1975) Giant-cell tumor of the radius treated by massive resection and tibial bone graft. Journal of Bone and Joint Surgery, 57-A, 982-986.

Crock, H. V. (1967) The Blood Supply of the Lower Limb Bones in Man. Edinburgh and London: E \& S Livingstone Ltd.

Dahlin, D. C., Cupps, R. E., and Johnson, E. W., Jun. (1970) Giant-cell tumor: a study of 195 cases. Cancer, 25, $1061-1070$.

Gold, A. M. (1957) Use of a prosthesis for the distal portion of the radius following resection of a recurrent giant-cell tumor. Journal of Bone and Joint Surgery, 39-A, 1374-1380.

Gold, A. M. (1965) Use of a prosthesis for the distal portion of the radius following resection of a recurrent giant-cell tumor. Journal of Bone and Joint Surgery, 47-A, 216-218.

Goldenberg, R. R., Campbell, C. J., and Bonfiglio, M. (1970) Giant-cell tumor of bone. An analysis of two hundred and eighteen cases. Journal of Bone and Joint Surgery, 52-A, 619-664.

Higinbotham, N. L., and Coley, B. L. (1950) The treatment of bone tumors by resection and replacement with massive grafts. Instructional Course Lectures, American Academy of Orthopaedic Surgeons, 7, 26-33.

Lawson, T. L. (1952) Fibular transplant for osteoclastoma of the radius. Journal of Bone and Joint Surgery. 34-B, 74-75.

McCullough, D. W., and Fredrickson, J. M. (1973) Neurovascular rib grafts to reconstruct mandibular defects. Canadian Journal of Otolaryngology, 2, 96-100.

Östrup, L. T., and Fredrickson, J. M. (1974) Distant transfer of a free, living bone graft by microvascular anastomoses. Plastic and Reconstructive Surgery, 54, 274-285.

Parrish, F. F. (1966) Treatment of bone tumors by total excision and replacement with massive autologous and homologous grafts. Journal of Bone and Joint Surgery, 48-A, 968-990.

Pho, R. W. H. (1979) Post-mortem studies of blood supply to the fibula using methylene blue and its clinical extension in living bone transplant. (In preparation.)

Pho, R. W. H., and Balachandran, N. (1978) Reversed proximal fibular graft in replacement of massive resection of lower end of radius in giant cell tumour. Paper presented at the 7th Singapore Orthopaedic Meeting.

Pitcock, J. A. (1971) Resection of distal radius and substitution by fibular transplant. In Campbell's Operative Orthopaedics. Fifth edition. Edited by A. H. Crenshaw. Vol. II, pp. 1425-1428. St Louis: C. V. Mosby Co.

Stewart, M. J., and Richardson, T. R. (1952) Giant-cell tumor of bone. Journal of Bone and Joint Surgery, 34-A, 372-386.

Taylor, G. I., Miller, G. D. H., and Ham, F. J. (1975) The free vascularized bone graft. Plastic and Reconstructive Surgery, 55, $533-544$.

Tuli, S. M. (1972) Bridging of bone defects by massive bone grafts in tumorous conditions and in osteomyelitis. Clinical Orthopaedics and Related Research, 87, 60-73.

Wilson, P. D., and Lance, E. M. (1965) Surgical reconstruction of the skeleton following segmental resection for bone tumors. Journal of Bone and Joint Surgery, 47-A, 1629-1656. 